\title{
Diagnostik und Therapie der Kolondivertikulitis
}

\author{
Diagnostics and Therapy of Colon Diverticulitis
}

\author{
Gesprächsleiter: G. Schürmann M. M. Lerch, Münster \\ Teilnehmer: R. Arbogast, Pforzheim R. Bittner, Stuttgart W. Domschke, Münster \\ T. Kocher, Basel, Schweiz A. Tuchmann, Wien, Österreich
}

\section{Einleitung}

Die Kolondivertikulitis ist auch heute noch ein herausforderndes Krankheitsbild, wobei die betroffenen Patienten primär sowohl dem Internisten/Gastroenterologen als auch dem Chirurgen zugewiesen werden. Die klinische Symptomatik ist mitunter uncharakteristisch und häufig durch Komorbidität verschleiert. Das diagnostische Armentarium wurde in den letzten Jahren erheblich erweitert, doch hat sich dadurch die Diagnosesicherheit erhöht? Diese und andere Fragen waren Gegenstand unseres (virtuellen) interdisziplinären Gesprächs mit eingeladenen Experten, kommentiert durch die Gesprächsleitung.

\section{Frage: Welche Untersuchungen sind zur Erhärtung der klinischen Verdachtsdiagnose Divertikulitis notwendig, und wann sollten sie durchgeführt werden? Welchen Stellenwert hat die Sonographie? Macht sie die Computertomographie (CT) entbehrlich?}

Kocher: Ist das klinische Bild klar, führen wir innerhalb von 24$48 \mathrm{~h}$ eine CT des Abdomens und Beckens mit oraler, rektaler und intravenöser Kontrastmittelgabe durch. Die Sonographie hat an unserer Klinik bei der initialen Beurteilung keine Bedeutung. Die Aussagekraft ist stark vom Untersucher abhängig, und eine differenzierte Beurteilung ist oft nicht möglich.

Arbogast: Neben der Klinik sollten zur Primärdiagnostik eine Abdomenübersichtsaufnahme (freie Luft?), ein Kolonkontrasteinlauf mit Gastrografin (ohne Luftinsufflation) und eine Sonographie durchgeführt werden. Der Stellenwert der Sonographie mit einer Sensitivität von $90 \%$ und einer Spezifität von $95 \%$ ist sehr untersucherabhängig und kann bei begleitender Paralyse und Adipositas in ihrer Aussagekraft gemindert sein, weshalb auf die CT aus meiner Sicht noch nicht verzichtet werden darf.

Tuchmann: Anamneseerhebung und klinische Untersuchung. La- bor: insbesondere Leukozyten, C-reaktives Protein. Bildgebende Diagnostik: Abdomenleeraufnahme, Sonographie, CT, Dickdarmeinlauf mit wasserlöslichem Kontrastmittel. Zur Feindiagnose verwenden wir die CT. Die Sonographie dient mehr der Übersicht.

Bittner: Bei klinischem Verdacht werden in angegebener Reihenfolge durchgeführt: 1. Labor, 2. Sonographie: Darstellung von wandverdickten Kolonabschnitten (Kokarde) und gasgefüllten Divertikeln oder von Abszeßformationen, 3. Röntgenübersichtsaufnahme des Abdomens zum Ausschluß freier Luft und eines Ileus, 4. Kolonkontrasteinlauf mit wasserlöslichem Kontrastmittel: Darstellung von Lokalisation und Ausmaß der Divertikulitis sowie möglichen Komplikationen wie Stenosierung, Fistelbildung und Perforation. Eine CT wird dann durchgeführt, wenn trotz Sonographie das Ausmaß der perikolischen entzündlichen Veränderungen nicht exakt bestimmt werden kann. Wenngleich die Sonographie überall zur Verfügung steht, wird die Interpretation des sonographischen Befundes erheblich von der Erfahrung des Untersuchers und den Untersuchungsbedingungen (Meteorismus, Koprostase) beeinflußt. Insofern kann die Sonographie die CT nicht vollständig ersetzen.

Domschke: Die Diagnose der akuten Divertikulitis basiert auf der Klinik des Patienten, ergänzt durch Laborbestimmungen der Entzündungsparameter (Blutbild, C-reaktives Protein). Initial besteht die Notwendigkeit zur Durchführung einer Abdomenübersichtsaufnahme zum Ausschluß einer freien Perforation, da diese eine umgehende chirurgische Intervention erzwingt. Der abdominale Ultraschall ist trotz seiner berichteten hohen Sensitivität und Spezifität stark abhängig von der Erfahrung des Untersuchers. Bei begleitender Darmparalyse wird seine Aussagekraft deutlich reduziert. Entsprechend läßt sich die CT nicht komplett durch den Ultraschall ersetzen.

Kommentar: Für die Initialdiagnostik sollte die klinische Untersuchung, unterstützt von Laboruntersuchungen (Entzündungsparameter) und der kompetenten Sonographie, im Vordergrund stehen. Eine Röntgenübersichtsaufnahme des Abdomens sollte zum Aus-

$\begin{array}{ll}\text { KARGER } & \text { @ 2000 S. Karger GmbH, Freiburg } \\ \text { Fax +497614520714 } & \text { Accessible online at: } \\ \begin{array}{l}\text { E-mail Information@Karger.de } \\ \text { www.karger.com }\end{array} & \text { www.karger.com/journals/cga }\end{array}$


schluß freier Luft erfolgen. Der Kolonkontrasteinlauf (mit wasserlöslichem Kontrastmittel) ist bei eindeutiger klinischer Symptomatik initial nicht obligat, sondern kann in der Regel frühelektiv nach 3-5 Tagen durchgeführt werden. Die CT kann vornehmlich differentialdiagnostische Überlegungen erhärten oder ausschließen. Sie steht bei der Initialdiagnostik im Hintergrund.

\section{Frage: Welche Komponenten sollte ein konservatives Therapieschema bei Patienten mit akuter Divertikulitis beinhalten? Nennen Sie Ihre Antibiotika der 1. und 2. Wahl?}

Kocher: Kurzzeitige Nahrungskarenz und i.v. Antibiotikagabe für 7-10 Tage. Bei raschem Ansprechen Wechsel auf perorale Gabe. Ambulante Weiterbetreuung in der Regel durch den Hausarzt. Stuhlregulation mit Quellmitteln (Methamucil, Colosan mite oder ähnliches). Antibiotikum der 1. Wahl: Amoxycyllin und Clavulansäure, Antibiotikum der 2. Wahl: Piperacillin und Tazobactam oder Meropenem.

Arbogast: Zunächst Nulldiät, dann ballaststoffarme Kost und bei Vorhandensein von Entzündungsparametern bzw. Fieber antibiotische Therapie. Antibiotika der 1. und 2. Wahl sind: Gyrasehemmer, Augmentan ${ }^{\circledR}$ (Amoxycyllin und Clavulansäure mit Metronidazol).

Tuchmann: Nahrungskarenz, Infusionstherapie, bei längerer Dauer parenterale Ernährung über zentralvenösen Zugang, Antibiotika: Clavulansäure/Amoxycyllin und Metronidazol oder Cephalosporin der zweiten Generation und Metronidazol. Bei Nichtansprechen sollte eine chirurgische Therapie in Erwägung gezogen werden, z. B. eine interventionelle Abszeßdrainage.

Bittner: Konservative Therapie einer akuten Divertikulitis beinhaltet zunächst die absolute Nahrungs- und Flüssigkeitskarenz und adäquate Infusionstherapie. Die intravenöse Breitspektrumantibiotika-Therapie wird primär mit Amoxycyllin/Clavulansäure und Metronidazol durchgeführt. Als Antibiotikum der 2. Wahl setzen wir Levofloxacin ein. Bei rückläufigen klinischen, laborchemischen und sonographischen Entzündungsbefunden wird der Kostaufbau mit diätetischer und medikamentöser Stuhlregulation eingeleitet.

Domschke: Bei Zeichen der akuten Divertikulitis ohne freie Perforation stellen die komplett parenterale Ernährung zur Entlastung des Darms, eine adäquate Schmerztherapie sowie die antibiotische Behandlung, die das Spektrum der Anaerobier einschließt, die Basis der Therapie dar. Acylaminopenicilline mit Betalaktamasen-Inhibitor bzw. Cephalosporine der Gruppe 3 in Kombination mit Metronidazol sehen wir als Mittel der 1. Wahl; bei Unverträglichkeit der Betalaktame bieten die Fluorchinolone der Gruppen 2 und seit neuestem auch 4 eine suffiziente Alternative. In Sonderfällen (Relaps, bekannte komplexe Resistenzlage) ergibt sich die Indikation zum Einsatz der Carbapeneme.
Kommentar: Die Vorschläge zur Therapie bieten wenig Platz für Kontroversen. Unabdingbar sind die Nahrungskarenz und der sofortige Einsatz von Antibiotika. Wegen der häufigen Mischinfektionen sollte eine Breitspektrumantibiose, die auch Anaerobier einschließt, vorgezogen werden.

\section{Frage: Wann erwägen Sie ein chirurgisches Vorgehen bei Patienten mit Divertikulitis (1. Episode) und in welchem Umfang spielen Begleitumstände (Alter des Patienten, Medikation, Komorbidität) eine Rolle bei Ihren Überlegungen?}

Kocher: Hinchey-Stadium II qualifiziert für frühelektive Chirurgie. Spezielle Patientensituation rechtfertigt manchmal spezielle (= unübliche) Vorgehensweise. Ansonsten haben wir unser Vorgehen detailliert beschrieben [1].

Arbogast: Wenn bei dem Patienten nachgewiesenermaßen eine 1. Episode der Divertikulitis vorliegt und die Untersuchungen wie Sonographie bzw. CT keine «komplizierte» Divertikulitis erkennen lassen, so ist ein chirurgisches Vorgehen noch nicht indiziert. Insbesondere dann nicht, wenn durch die gewählte konservative Therapie sich das Beschwerdebild rasch zurückbildet, denn dies sind bekanntermaßen die «leichteren Fälle». Je jünger allerdings der Patient bzw. je umfangreicher die Begleiterkrankungen sind, um so früher sollte die primäre Resektion angestrebt werden, um den 2. Schub mit in der Regel gravierenderen Komplikationen zu vermeiden.

Tuchmann: Beim 2. oder 3. Divertikulitisschub denken wir an eine chirurgische Therapie. Beim ersten Schub operieren wir nur dann, wenn Therapieresistenz besteht oder ein Karzinomverdacht vorliegt. Deswegen koloskopieren wir alle Patienten nach Rückgang der klinischen Divertikulitissymptomatik etwa 10 Tage nach Beginn der Erkrankung. Begleitumstände spielen selbstverständlich eine Rolle sowie die persönlichen Referenzen unserer Patienten.

Bittner: Die Indikation zur Operation ist in jedem Fall gegeben, wenn Komplikationen der Divertikulitis vorliegen. Hier ist die Indikationsstellung weitgehend unabhängig von Alter oder Komorbidität der Patienten. In einer solchen Situation muß der septische Herd sofort beseitigt werden. Bei rezidivierenden Divertikulitisepisoden ohne Komplikationen ist eine elektive Kolonresektion im beschwerdefreien Intervall zu erwägen. Analysen unseres Patientenkollektivs haben gezeigt, daß es offenbar zwei verschiedene Verlaufsformen der Divertikulitis gibt. Heftige Entzündungsbefunde mit Perforationen haben oft eine relativ kurze Anamnese und werden häufig im Rahmen einer 1. Entzündungsepisode diagnostiziert, während chronisch rezidivierende Entzündungsepisoden eher selten in einer Notfallsituation mit Perforation münden. Bei Patienten $<60$ Jahre ohne schwerwiegende Begleiterkrankungen empfehlen wir die elektive Operation im allgemeinen erst nach mehreren (2) Divertikulitisepisoden nach Ausschöpfen der pro- 
phylaktischen Maßnahmen bei Diät und Lebensgewohnheiten. Ältere Patienten mit Begleiterkrankungen weisen bei der Operation einer komplizierten Divertikulitis eine hohe Morbidität auf, so daß bei diesen Patienten die Operationsindikation eher zu stellen ist, da diese bei einer möglichen Progredienz der Erkrankung extrem gefährdet sind.

Domschke: Ein chirurgisches Vorgehen im Rahmen der akuten Entzündungsphase steht bei Zeichen der freien Perforation im Vordergrund. Auch ein fehlendes Ansprechen auf die konservative Therapie mit septischen Komplikationen stellt eine Indikation zur umgehenden operativen Behandlung dar. Die Entscheidung zur Therapieumstellung (d.h. zur Operation) sollte gerade bei Patienten mit geringeren physiologischen Reserven (Komorbidität, höheres Alter) großzügiger gestellt werden.

Kommentar: Der 1. Schub einer «unkomplizierten» Divertikulitis wird auch in Münster konservativ behandelt. In der Regel tritt eine Besserung der klinischen Symptomatik spätestens nach 2-3 Tagen nach den erwähnten therapeutischen Maßnahmen ein. Allerdings ist unser Patientenkollektiv durch die für eine Universitätsklinik typische hohe Komorbidität gekennzeichnet. Viele unserer Patienten haben eine attenuierte klinische und laborchemische Symptomatik, so z. B. unsere unter Immunsuppression stehenden transplantierten Patienten. Insbesondere bei letztgenannter Patientengruppe setzen wir die Hürde zur chirurgischen Therapie deutlich niedriger als bei Patienten ohne Komorbidität.

\section{Frage: Welches operative Verfahren wählen Sie bei nachgewiesener Divertikelperforation? Was sind gegebenenfalls Ihre Entscheidungskriterien für die Anwendung verschiedener operativer Verfahren, insbesondere für Ihre Entscheidung zwischen Kontinuitäts- und Diskontinuitätsresektion?}

Kocher: Lediglich bei Patienten mit kotiger Peritonitis (HincheyStadium IV) oder zusätzlichen Risikofaktoren (Immunsuppression, schlechter Ernährungszustand, Sepsis oder andere Risikofaktoren) ist eine Diskontinuitätsresektion nach Hartmann noch indiziert. Alle anderen Patienten erhalten eine Primäresektion mit Primäranastomose (oft in Verbindung mit On-table-Lavage). Verfahren ohne Resektion des Entzündungsherdes (alleinige Kolostomie oder Drainage) sind mit hoher Morbidität und Letalität behaftet. Die Indikation zu diesem Vorgehen ist polymorbiden Patienten vorbehalten, denen ein größerer Eingriff nicht zugemutet werden kann.

Arbogast: Auch bei der nachgewiesenen Divertikelperforation und einer Peritonitis in den Stadien III und IV nach Hinchey entscheiden wir uns nahezu ausnahmslos zur primären Resektion mit primärer Anastomose. Diese Patienten mit in der Regel einer 4-Quadranten-Peritonitis werden in die programmierte Lavage aufgenommen und so mit einer Anastomose im 24-Stunden-Rhythmus inspiziert und kontrolliert. Für ein mehrzeitiges Vorgehen, so insbesondere die Operation nach Hartmann, besteht nach meiner Ansicht praktisch keine Indikation mehr. Auch bei primärer Resektion ist die Anlage eines Anus praeter überflüssig.

Tuchmann: Bei nachgewiesener Divertikelperforation wird unverzüglich laparotomiert. Wir streben eine primäre Resektion und Anastomosierung, unabhängig vom Hinchey-Stadium, an. Voraussetzung für uns sind On-table-Lavage, gut durchblutete, nicht entzündlich veränderte Darmabschnitte für die Anastomose und erträglicher Patientenzustand bzw. Komorbidität. Die Operation erfolgt nach Rücksprache mit dem Anästhesisten. In allen anderen Fällen sowie bei instabilen Patienten kommt die Diskontinuitätsresektion zur Anwendung.

Bittner: Das Prinzip der chirurgischen Therapie besteht aus der Sanierung des entzündlichen Herdes, d.h. Resektion des entzündlich veränderten Kolonsegments, gegebenenfalls mit Ausräumung eines Abszesses oder der Lavage der Bauchhöhle. Unseres Erachtens ist dies immer erforderlich und möglich. Unsere Erfahrungen zeigen weiterhin, daß auch bei einer Notfalloperation wegen Perforation und lokaler Peritonitis, also bei komplizierter Divertikulitis, eine Anastomosierung und somit eine sofortige Wiederherstellung der Darmpassage sicher möglich ist und deshalb angestrebt werden kann. Bei schwieriger Anastomose sollte ein protektiver doppelläufiger Anus praeter (oder Ileostoma) vorgeschaltet werden. Die Alternative stellt die Diskontinuitätsresektion nach Hartmann dar. Dieses Verfahren ist auch heute noch sinnvoll, wenn z. B. bei diffuser Peritonitis eine Anastomosierung aufgrund entzündlich veränderter oder schlecht perfundierter Resektionsränder (oder in nächtlicher Situation unerfahrener Chirurg) nicht sicher möglich ist. Diese Situation liegt in unserem Krankenkollektiv jedoch nur bei $<5 \%$ der Fälle vor.

Kommentar: Wer bei schwerer Divertikulitis die kontinenzerhaltende Resektion ohne Stomaanlage par principe propagiert, nimmt zumindest bei einem Teil der Patienten ein hohes Risiko in Kauf. Die hohe Prävalenz von Komorbidität und insbesondere Immunsuppression in unserem Patientenkollektiv, wie angesprochen, bedingt bei unseren Patienten gelegentlich auch die Diskontinuitätsresektion oder die Anlage eines protektiven Stomas. Auch bei uns wird bei 4-Quadranten-Peritonitis die programmierte Relaparotomie und Lavage durchgeführt. Welche Vorgehensweise auch immer gewählt wird, oberstes Ziel sollten die Verfahrenssicherheit und Vermeidung einer Anastomoseninsuffizienz sein, die insbesondere bei multimorbiden Patienten mit einer hohen Mortalitätsrate verbunden sind. 


\section{Frage: Wie definieren Sie eine «therapierefraktäre Divertikulitis", und welche Konsequenzen ziehen sie aus dieser Diagnose?}

Kocher: Kenne ich nicht. Gibt es das? Inadäquate konservative Therapie? Andere Pathologie (Reizdarmsyndrom)?

Arbogast: Die Definition der sogenannten therapierefraktären Divertikulitis ist ausgesprochen schwierig. Ist die Divertikulitis therapierefraktär, so ist die Resektion des Fokus unstreitig indiziert. Kommt es jedoch nach Sigmaresektion zu einem Divertikulititsrezidiv, was jedoch in deutlich $<4 \%$ der Fälle zu erwarten ist, so muß in seltenen Fällen einer erneute Resektion des entzündeten Darmabschnitts durchgeführt werden. Da jedoch der Ausgangspunkt der Divertikulitis in fast allen Fällen die Hochdruckzone im rektosigmoidalen Übergangsbereich ist und dieser im Regelfall reseziert worden sein sollte, so ist mit einem echten Divertikulititsrezidiv nur dann zu rechnen, wenn das Resektionsausmaß nach distal beim Primäreingriff nicht ausreichend war.

Tuchmann: Therapierefraktär heißt Befundverschlechterung bzw. keine Besserung der Erkrankung nach einer Woche. In einem solchen Fall würden wir die Diagnostik wiederholen, eventuell CT-gezielte Abszeßdrainage. Die nächste Konsequenz wäre die Operation.

Bittner: Eine therapierefraktäre Divertikulitis liegt dann vor, wenn trotz Ausschöpfung der konservativen, prophylaktischen und therapeutischen Maßnahmen weiterhin ein Entzündungsbefund zu erheben ist bzw. rezidivierende Entzündungsepisoden $(>2)$ beobachtet werden. In diesem Fall besteht die Indikation zur Kolonresektion.

Domschke: Sollten nach $48 \mathrm{~h}$ eines optimalen konservativen Managements keine klinischen Zeichen der Besserung erkennbar sein, so müßte eine Schnittbilddiagnostik im Sinne eines CT zum Nachweis eines Abszesses durchgeführt werden. Eine präoperative perkutane Abszeßdrainage kann das Ergebnis einer späteren Operation günstig beeinflussen.

Kommentar: Die therapierefraktäre Divertikulitis kann von dem Divertikulitisrezidiv klar differenziert werden. Eine therapierefraktäre Divertikulitis liegt vor, wenn trotz konservativer Therapie (siehe oben) die klinische Symptomatik persistiert. Insbesondere bei Risikopatienten (Patienten mit attenuierter klinischer Symptomatik) stellen wir die Operationsindikation bereits nach 23 Tagen.

\section{Frage: Welche Empfehlungen geben Sie den Patienten nach erfolgreich behandeltem 1. Schub einer Divertikulitis? Was sind Ihre Überwachungsstrategien?}

Kocher: Stuhlregulation und ausreichende Flüssigkeitszufuhr. 46 Wochen nach Entzündungsschub wird koloskopiert. Keine spezifische Überwachung nötig. Was soll man überwachen? Ergeben sich durch die Überwachung irgendwelche Konsequenzen? Nein, sie kostet Zeit und Geld. Der aufgeklärte Patient meldet sich bei erneutem Entzündungsschub.

Arbogast: Diesen Patienten empfehlen wir körperliche Bewegung, vor allen Dingen nach dem Abendessen, ballaststoffreiche Kost, reichlich Flüssigkeit und weisen sie nachhaltig darauf hin, daß sie sich selbst bei geringer Symptomatik im linken Mittel- und Unterbauch sofort zum Chirurgen begeben sollten. Weitere wesentliche Überwachungsstrategien sind unseres Erachtens nicht erforderlich, sie sei denn, daß bei den Patienten sekundäre Probleme wie Stenosen und Fistelungen (Pneumaturie) auftreten.

Tuchmann: Zur Rezidivprophylaxe nach konservativer Therapie empfehlen wir eine ausgewogene Kost, reichlich Flüssigkeit, reichlich körperliche Bewegung, vorsichtige Ballaststofferhöhung und Gewichtsreduktion. Unter diesen Maßnahmen sollte sich der intrakolonische Druck reduzieren, der Patient soll auf täglichen Stuhlgang achten. Diesbezügliche Kontrollen erfolgen in der Sprechstunde, wahlweise im Krankenhaus oder beim niedergelassenen Arzt.

Bittner: Alle Divertikulitispatienten werden über die Ursache und die Komplikationsmöglichkeiten der Erkrankung aufgeklärt. Des weiteren erfolgt eine Ernährungsberatung durch eine geprüfte Diätassistentin. Prinzipiell werden eine ballaststoffreiche Kost und eine gesteigerte Flüssigkeitszufuhr empfohlen. Zur Überwachung sind regelmäßige klinische, laborchemische und sonographische Untersuchungen sinnvoll. Bereits nach Abklingen der ersten Entzündungsepisode sollte eine Koloskopie durchgeführt werden, da diese Untersuchung die höchste Sensitivität zur Diagnostik von entzündlichen und neoplastischen Schleimhautveränderungen des Kolons aufweist. So können andere Erkrankungen wie chronisch entzündliche Darmerkrankungen oder Polypen bzw. Karzinome zuverlässig ausgeschlossen und diagnostiziert werden.

Domschke: Bei erfolgreich kontrollierter Entzündung empfiehlt sich die Einhaltung einer ballaststoffreichen Diät, ausreichende Flüssigkeitszufuhr sowie körperliche Bewegung und Gewichtsreduktion. In diesen Phasen sollte eine endoskopische Diagnostik zur Dokumentation von Befallsmuster und -ausdehnung, aber auch zum Ausschluß eines synchron vorliegenden Kolonkarzinoms erfolgen. Die weitere Betreuung sollte symptomorientiert sein; die Überwachung dieser Patienten beinhaltet die übliche Tumorvorsorge sowie eine umgehende endoskopische Diagnostik zum Ausschluß eines kolorektalen Karzinoms bei einer Veränderung des Beschwerdebildes. 
Kommentar: Sofern nicht bereits nach dem 1. Divertikulitisschub geschehen, sollte das Intervall auf jeden Fall für eine adäquate Kolondiagnostik genutzt werden. Hier scheint uns ein Zeitintervall von 4-6 Wochen sinnvoll. Ballaststoffreiche Kost und gute Stuhlregulation sollten angestrebt werden. Bei gründlicher Aufklärung und Compliance des Patienten sind programmierte Überwachungsuntersuchungen in der Regel nicht erforderlich. Der überwiegende Teil der Patienten bleibt nach einer 1. Divertikulitisepisode rezidivfrei.

\section{Frage: 2. Schub der Divertikulitis: Sollte man sofort, frühelektiv nach Abklingen der Entzündungssymptomatik, im freien Intervall operieren oder, in Abhängigkeit vom Risikoprofil des Patienten, weitere Schübe abwarten?}

Kocher: Je nach Hinchey-Stadium wird frühelektiv oder elektiv operiert. Je nach Patientensituation (Alter, Komorbidität) und in Abhängigkeit von den entzündlichen Veränderungen kann eine abwartende Haltung angezeigt sein.

Arbogast: Der 2. Schub einer Divertikulitis (dieser ist meist schon der 3. oder 4.) sollte Anlaß sein, so rasch wie möglich die elektive Resektion durchzuführen. Dies sollte während des gleichen stationären Aufenthalts nach primär über wenige Tage andauernder konservativer Behandlung nach Abklingen der Entzündungssymptomatik erfolgen. Unsere Erfahrungen mit den Patienten, die nach dem 2. Schub mit der Maßgabe nach Hause entlassen wurden, daß im freien Intervall die Resektion durchgeführt werden sollte, kommen entweder gar nicht oder dann im akuten 3. oder weiteren Schub. Je höher das Risikoprofil der Patienten ist, um so früher sollte man sich zur definitiven Fokussanierung entscheiden. Da diese Patienten mit einer Wahrscheinlichkeit von $80 \%$ weitere Schübe erleiden, die dann vielleicht mit einer freien Perforation einhergehen, werden diese Patienten in eine deutlich schlechtere Ausgangssituation gebracht. Bei ihnen liegt die Letalitätsquote um $10 \%$.

Tuchmann: Wir stellen die Operationsindikation nach dem 2. oder 3. Schub, frühelektiv nach Abklingen der Entzündungssymptomatik. Es besteht jedoch eine große Variabilität, bedingt durch Komorbidität sowie Patientencompliance. Es gibt auch Patienten, die kurze und leicht behandelbare Divertikulitisepisoden haben und über den 3. Schub hinaus konservativ behandelt werden. Viele dieser Patienten kommen wahrscheinlich gar nicht ins Krankenhaus.

Bittner: Diese Frage wird weitgehend bereits bei Frage 3 diskutiert. Aus unserer Sicht besteht nach dem 2. Schub nicht prinzipiell die Indikation zur Operation. Diese muß immer individuell gemeinsam mit dem Patienten unter Berücksichtigung der Intensität der stattgehabten Entzündungsepisoden, der Lebenssituation und der Wünsche und Ängste des Patienten erarbeitet werden. Falls nach Einleitung einer konservativen Therapie innerhalb weniger Tage ein Rückgang der Entzündungssymptomatik und eine Stabilisierung des Allgemeinzustands zu beobachten ist, so kann die Operation im beschwerdefreien Intervall erfolgen. Kommt es jedoch nach Ausschöpfen der konservativen Therapiemaßnahmen nicht zur raschen Besserung, so sollte nicht lange mit der Operation gezögert werden, um den Patienten nicht unnötig zu gefährden und die Krankheitsepisode zu verlängern.

Domschke: Eine operative Therapie im Rahmen des akuten Schubs sollte bei Zeichen der freien Perforation oder eines unter konservativen Behandlung therapierefraktären Verlaufs erfolgen. Sollte die konservative Therapie beim Rezidiv der Divertikulitis zum Erfolg führen, so wäre eine frühelektive Sanierung zu empfehlen, zumal dann die notwendige Motivation des Patienten vorhanden sein dürfte. Bei einem längeren ambulanten Intervall finden sich viele der Patienten nicht vor einem erneuten Entzündungsschub beim Chirurgen ein.

Kommentar: Der 2. Entzündungsschub einer Kolondivertikulitis sollte in der Regel zur Vereinbarung einer operativen Sanierung führen. Die Operation sollte im Intervall erfolgen, wobei unseres Erachtens der Patient auch zwischenzeitlich entlassen werden kann. Bei guter Patientenführung sollte die stationäre Wiederaufnahme zur frühelektiven operativen Sanierung kein Problem sein. Diese Vorgehensweise ist insbesondere bei hoher Komorbidität im Hinblick auf eine attenuierte klinische Symptomatik (siehe oben) erstrebenswert. Wir weichen von dieser standardisierten Vorgehensweise nur in Fällen mit deutlich erhöhtem Operationsrisiko oder bei Vorliegen sonstiger Begleitumstände ab, die gegenüber der Operationsindikation beim 2. Schub der Sigmadivertikulitis zu beachten sind. Eine sofortige Operation ist selten erforderlich hier gelten im wesentlichen die gleichen Indikationskriterien wie beim 1. Schub. Die Bevorzugung eines operativen Vorgehens nach dem 2. Schub einer Divertikulitis basiert in erster Linie auf der statistischen Beobachtung, daß nach diesem, anders als nach dem 1. Ereignis, die Rezidivwahrscheinlichkeit sehr viel höher ist.

\section{Frage: Welche (erneuten) diagnostischen Maßnahmen führen Sie vor einer geplanten Operation im freien Intervall nach Divertikulitis durch?}

\section{Kocher: Keine.}

Arbogast: Erfolgt die Operation im freien Intervall relativ früh, d.h. innerhalb von 3 Monaten nach dem letzten Divertikulitisereignis, so sind außer einer Sonographie auch aus unserer Sicht keine weiteren diagnostischen Maßnahmen indiziert. Liegt das Intervall jedoch länger als 6 Monate zurück, so empfehlen wir neben der Sonographie bzw. einer CT-Diagnostik erneut einen Kolonkontrasteinlauf, der im wirklich freien Intervall zur Feindiagnostik auch mit Barium durchgeführt werden kann, bzw. die Koloskopie. 
Tuchmann: Vor einer geplanten Operation im Intervall werden alle diagnostischen Maßnahmen (Frage 1) wiederholt. Statt der Kontrastmitteluntersuchung wird eine präoperative Koloskopie zum Karzinomausschluß durchgeführt.

Bittner: Wie vor jeder geplanten abdominalchirurgischen Operation sollten die Basisdiagnostik, die Laboruntersuchungen und die Sonographie des Abdomens durchgeführt werden. Neben einem aktuellen Kolonkontrasteinlauf erfolgt eine Koloskopie zur Befunddokumentation bzw. zum Ausschluß weiterer Erkrankungen des Kolons.

Domschke: Beim umgehenden Anschluß der Operation an die konservative Behandlung des akuten Divertikulitisschubs bedarf es in der Regel keiner erneuten Bildgebung. Ansonsten wäre eine Schnittbilddiagnostik im Sinne einer CT ratsam. Bei zuvor noch nicht koloskopierten Patienten müßte eine endoluminale Diagnostik (möglichst Endoskopie, gegebenenfalls Kolonkontrasteinlauf) unter den Gesichtspunkten einer Beschreibung des Ausdehnungsmusters sowie des Ausschlusses synchron vorliegender Karzinome erfolgen.

Kommentar: Wurde die übliche Kolondiagnostik (Koloskopie und/ oder Kolonkontrasteinlauf) bereits nach dem ersten Entzündungsschub im symptomfreien Intervall durchgeführt, so wiederholen wir diese Maßnahmen nicht grundsätzlich vor dem jetzt anstehenden operativen Eingriff, es sei denn, die längere Wartezeit oder eine veränderte Symptomatik ergeben Hinweise auf eine neue Diagnose oder einen Zusatzbefund.

\section{Literatur}

1 Kocher T, Remmel E, Harder F: Abklärung und Therapie der linksseitigen Kolondivertikulitis - Schweizer Erfahrungen. Chir Gastroenterol 2000;16:332.

\section{Teilnehmer}

Prof. Dr. R Arbogast

Chirurgische Klinik

Städtisches Klinikum Pforzheim

D-75116 Pforzheim

Tel +49 7231 969-286, Fax -670

E-mail Arbogast.Chirurgische_Klinik@Pforzheim.de

Prof. Dr. R. Bittner

Klinik für Allgemein- und Visceralchirurgie

Marienhospital

Böheimstraße 37

D-70199 Stuttgart

Tel. +49 7116489 22-01, Fax -13

E-mail reinhardbittner@vinzenz.de
Prof. Dr. W. Domschke

Medizinische Klinik und Poliklinik B Westfälische Wilhelms-Universität Münster

Albert-Schweitzer-Straße 33

D-48129 Münster

Tel. +49 251 83-47 661, Fax -570

E-mail

Dr. T. Kocher

Allgemeinchirurgische Klinik

Departement Chirurgie

Universität Basel

Kantonsspital Basel

CH-4031 Basel

Tel. +41 61 265-25 25, Fax -77 92

E-mail Thomas.Kocher@unibas.ch
Prof. Dr. A. Tuchmann

Chirurgische Abteilung

Krankenhaus Floridsdorf

Hinaysgasse 1

A-1210 Wien

Tel. +43 1275 22-0, Fax -4109 